\title{
Total Synthesis of the Marine Natural Product (-)-Clavosolide A.
}

\section{A Showcase for the Petasis-Ferrier Union / Rearrangement Tactic}

Amos B. Smith, III and Vladimir Simov

Department of Chemistry, Monell Chemical Senses Center, and Laboratory for Research on the Structure of Matter, University of Pennsylvania, Philadelphia, Pennsylvania 19104, U.S.A.

Supporting Information 
Materials and Methods: All solvents were reagent grade. Diethyl ether $\left(\operatorname{Et}_{2} \mathrm{O}\right)$ and tetrahydrofuran (THF) were freshly distilled from sodium/benzophenone under argon, while dichloromethane $\left(\mathrm{CH}_{2} \mathrm{Cl}_{2}\right)$ was distilled from calcium hydride $\left(\mathrm{CaH}_{2}\right)$. All reagents were purchased from Aldrich or Acros and used as received. Crotonaldehyde was distilled over calcium hydride $\left(\mathrm{CaH}_{2}\right)$ before each aldol reaction. Dicyclohexylboron triflate was freshly prepared ${ }^{1}$ and stored in hexanes under argon at $0{ }^{\circ} \mathrm{C}$. Reactions were magnetically stirred under an argon atmosphere and monitored by thin layer chromatography (TLC) with $0.25 \mathrm{~mm}$ E. Merck precoated silica gel plates. Flash chromatography was performed with silica gel 60 (particle size $0.040-0.062$ $\mathrm{mm}$ ) supplied by Silicycle and Sorbent Technologies. Yields refer to chromatographically and spectroscopically pure compounds, unless otherwise stated. Infrared spectra were recorded on a Jasco Model FT/IR-480 Plus spectrometer. Proton and carbon-13 NMR spectra were recorded on a Bruker AMX-500 spectrometer. Chemical shifts are reported relative to either chloroform $(\square 7.27)$ or benzene $(\square 7.16)$ for ${ }^{1} \mathrm{H}$ NMR and either chloroform $(\square 77.2)$ or benzene $\left(\square\right.$ 128.4) for ${ }^{13} \mathrm{C}$ NMR. Optical rotations were measured on a Perkin-Elmer model 241 polarimeter. High resolution mass spectra were measured at the University of Pennsylvania Mass Spectrometry Service Center.

\section{Experimental Section.}

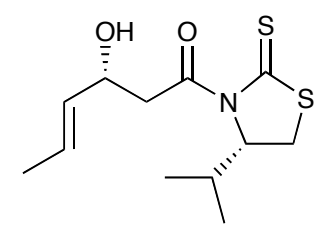

$(+)-9$

Allylic alcohol (+)-9. To a $0^{0} \mathrm{C}$ solution of Nagao auxillary (+)-8 (8.56 g, $\left.1.70 \mathrm{eq}\right)$ in $\mathrm{CH}_{2} \mathrm{Cl}_{2}(290 \mathrm{~mL})$ was added $\mathrm{TiCl}_{4}\left(47.1 \mathrm{~mL}, 1.0 \mathrm{M}\right.$ in $\left.\mathrm{CH}_{2} \mathrm{Cl}_{2}, 1.90 \mathrm{eq}\right)$ and the resulting solution was stirred for 5 min. before cooling to $-78^{\circ} \mathrm{C}$. DIPEA ( $N, N$-Diisopropylethylamine) $(8.19 \mathrm{~mL}, 1.90 \mathrm{eq})$ was then added dropwise, and the resulting enolate solution was stirred for $2 \mathrm{~h}$ at $-78^{\circ} \mathrm{C}$. Crotonaldehyde $(2.02 \mathrm{~mL}, 24.7 \mathrm{mmol}) \mathrm{in} 80 \mathrm{~mL}$ $\mathrm{CH}_{2} \mathrm{Cl}_{2}$ was then added dropwise to the reaction mixture, and the resulting solution stirred at $-78{ }^{\circ} \mathrm{C}$ for an additional $2 \mathrm{~h}$. The reaction was quenched with $100 \mathrm{~mL}$ of saturated $\mathrm{NH}_{4} \mathrm{Cl}$ solution, diluted with $200 \mathrm{~mL}$ $\mathrm{CH}_{2} \mathrm{Cl}_{2}$ and allowed to warm to RT. The layers were separated and the aqueous layer was extracted with $\mathrm{CH}_{2} \mathrm{Cl}_{2}(100 \mathrm{~mL}, 3 \mathrm{x})$. The combined organic layers were then washed with $\mathrm{H}_{2} \mathrm{O}(100 \mathrm{~mL})$ and brine (100 $\mathrm{mL}$ ), dried over $\mathrm{MgSO}_{4}$ and concentrated. Flash chromatography (5:1 to 1:1 = hex:EtOAc) afforded allylic alcohol (+)-9 (6.36 g, 94\% yield). [ [ $]_{\mathrm{D}}{ }^{20}+347.5$ (c 2.10, $\mathrm{CHCl}_{3}$ ); IR (thin film, $\left.\mathrm{CDCl}_{3}\right) 3427$ (br, w), 2962 (m), 1693 (s), 1364 (m), 1167 (s), 1039 (m) cm ${ }^{-1} ;{ }^{1} \mathrm{H}$ NMR (500 MHz, $\mathrm{CDCl}_{3}$ ) $\square .76$ (dq, J = 6.3, 15.6 Hz, $1 \mathrm{H}), 5.56(\mathrm{dd}, J=6.3,15.6 \mathrm{~Hz}, 1 \mathrm{H}), 5.16(\mathrm{t}, J=7.5 \mathrm{~Hz}, 1 \mathrm{H}), 4.62(\mathrm{br} \mathrm{t}, J=6.0 \mathrm{~Hz}, 1 \mathrm{H}), 3.61(\mathrm{dd}, J=2.6$, $17.9 \mathrm{~Hz}, 1 \mathrm{H}), 3.53(\mathrm{dd}, J=8.2,11.2 \mathrm{~Hz}, 1 \mathrm{H}), 3.30(\mathrm{q}, J=8.9 \mathrm{~Hz}, 1 \mathrm{H}), 3.04(\mathrm{~d}, J=11.9 \mathrm{~Hz}, 1 \mathrm{H}), 2.78$ (br s, 
$1 \mathrm{H},-\mathrm{OH}), 2.37(\mathrm{~m}, 1 \mathrm{H}), 1.72(\mathrm{~d}, J=6.3 \mathrm{~Hz}, 3 \mathrm{H}), 1.07(\mathrm{~d}, J=6.7 \mathrm{~Hz}, 3 \mathrm{H}), 0.99(\mathrm{~d}, J=6.7 \mathrm{~Hz}, 3 \mathrm{H}) ;{ }^{13} \mathrm{C}$ NMR $\left(125 \mathrm{MHz}, \mathrm{CDCl}_{3}\right) \square 202.9,172.5,131.7,127.3,71.3,68.7,45.4,30.8,30.6,19.0$, 17.7, 17.6; high resolution mass spectrum $(\mathrm{ES}+) \mathrm{m} / z, 296.0764$ [(M+Na) ${ }^{+}$; calcd for $\mathrm{C}_{12} \mathrm{H}_{19} \mathrm{O}_{2} \mathrm{NS}_{2} \mathrm{SiNa}^{+}$: 296.0755].

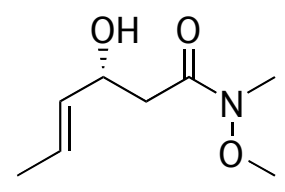

(+)-6

Weinreb amide (+)-6. To a solution of thione (+)-9 $(10.1 \mathrm{~g}, 37.1 \mathrm{mmol})$ in $\mathrm{CH}_{2} \mathrm{Cl}_{2}(190 \mathrm{~mL})$ was added imidazole (12.6 g, $5.0 \mathrm{eq})$ and $\mathrm{HN}\left(\mathrm{OCH}_{3}\right) \mathrm{CH}_{3}-\mathrm{HCl}(9.0 \mathrm{~g}, 2.50 \mathrm{eq}$, azeotroped 3x with benzene) and the resulting suspension was stirred at $\mathrm{RT}$ for $12 \mathrm{~h}$. The reaction was quenched with saturated $\mathrm{NH}_{4} \mathrm{Cl}$ solution $(120 \mathrm{~mL})$ and the layers were separated. The aqueous layer was then extracted with $\mathrm{CH}_{2} \mathrm{Cl}_{2}(200 \mathrm{~mL}, 3 \mathrm{x})$, and the combined organic layers were dried over $\mathrm{MgSO}_{4}$ and concentrated. Flash chromatography (2:1 then 0:1 = hex:EtOAc) afforded the recovered auxillary (>90\% recovery) and Weinreb amide (+)-6 (5.9 g, $92 \%$ yield). [ []$_{\mathrm{D}}^{20}+48.4$ (c 1.94, $\mathrm{CHCl}_{3}$ ); IR (thin film, $\left.\mathrm{CDCl}_{3}\right) 3424$ (br, m), 2941 (br, w), 1640 (s), 1441 (m), 1385 (m); ${ }^{1} \mathrm{H}$ NMR (500 MHz, $\left.\mathrm{CDCl}_{3}\right) \square 5.75(\mathrm{dq}, J=6.3,15.2 \mathrm{~Hz}, 1 \mathrm{H}), 5.54(\mathrm{dd}, J=6.3,15.2 \mathrm{~Hz}, 1 \mathrm{H})$, 4.50 (br t, $J=7.0 \mathrm{~Hz}, 1 \mathrm{H}), 3.68(\mathrm{~s}, 3 \mathrm{H}), 3.19$ (s, 3H), $2.64(\mathrm{~m}, 2 \mathrm{H}), 1.70(\mathrm{~d}, J=5.7 \mathrm{~Hz}, 3 \mathrm{H}) ;{ }^{13} \mathrm{CNMR}(125$ $\left.\mathrm{MHz}, \mathrm{CDCl}_{3}\right) \square 179.3,135.7,130.1,68.5,60.5,36.4,29.4,14.3$; high resolution mass spectrum $(\mathrm{ES}+) \mathrm{m} / \mathrm{z}$ $196.0955\left[(\mathrm{M}+\mathrm{Na})^{+}\right.$; calcd for $\mathrm{C}_{8} \mathrm{H}_{15} \mathrm{O}_{3} \mathrm{NNa}^{+}$: 196.0950$]$.

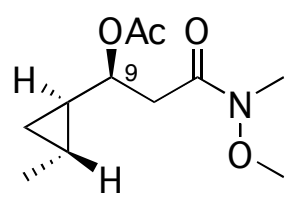

(-)-SI 1

Acetate (-)-SI 1. Allylic alcohol (+)-6 (3.01 g, $17.4 \mathrm{mmol})$ in $180 \mathrm{~mL} \mathrm{CH}_{2} \mathrm{Cl}_{2}$ was cooled to $-15{ }^{\circ} \mathrm{C}$ and treated dropwise with $\mathrm{Et}_{2} \mathrm{Zn}(8.91 \mathrm{~mL}, 5.0 \mathrm{eq}$, spontaneously flammable in air) dropwise. After 5 minutes, $\mathrm{CH}_{2} \mathrm{I}_{2}(7.03 \mathrm{~mL}, 5.0 \mathrm{eq})$ was added slowly, and the resulting solution was allowed to warm to RT over $3 \mathrm{~h}$. The reaction was then stirred for an additional $1 \mathrm{~h}$, quenched via the dropwise addition of a saturated $\mathrm{NH}_{4} \mathrm{Cl}$ solution $(80 \mathrm{~mL})$, diluted with $\mathrm{CH}_{2} \mathrm{Cl}_{2}(160 \mathrm{~mL})$, and then acidified with $10 \% \mathrm{HCl}(80 \mathrm{~mL})$. The layers were then separated and the aqueous layer was extracted with $\mathrm{CH}_{2} \mathrm{Cl}_{2}(200 \mathrm{~mL}, 2 \mathrm{x})$. The combined organic layers were then successively washed with saturated solutions of $\mathrm{Na}_{2} \mathrm{SO}_{3}, \mathrm{NaHCO}_{3}$ and brine $(120 \mathrm{~mL}$ each), dried over $\mathrm{MgSO}_{4}$ and concentrated. The crude syn-cyclopropylcarbinol (+)-10 was judged pure by NMR and used without further purification. 
To a $-45{ }^{\circ} \mathrm{C}$ solution of $\mathrm{PPh}_{3}(12.5 \mathrm{~g}, 2.7 \mathrm{eq})$ in toluene $(410 \mathrm{~mL})$ was added DIAD (diisopropyl azodicarboxylate) $(11.3 \mathrm{~mL}, 3.2 \mathrm{eq})$ and the resulting mixture stirred for 2 minutes before adding $\mathrm{AcOH}$ $(2.76 \mathrm{~mL}, 2.70 \mathrm{eq})$. The crude syn-cyclopropylcarbinol (+)-10 in toluene $(70 \mathrm{~mL})$ was then quickly added dropwise, and the resulting solution stirred at $-45{ }^{\circ} \mathrm{C}$ for $8 \mathrm{~h}$. Once completed, the reaction was concentrated and purified by flash chromatography $(3: 1=$ hex:EtOAc) to afford acetate (-)-SI 1 (2.95 g, 74\% yield, 2 steps) as an inseparable 12:1 mixture of C9 diastereomers. [ []$_{\mathrm{D}}{ }^{20}-26.1$ (c 1.31, $\mathrm{CHCl}_{3}$ ); IR (thin film, $\left.\mathrm{CDCl}_{3}\right) 3000$ (br, w), 1733 (s), 1667 (s), 1242 (s), 1020 (m) cm ${ }^{-1} ;{ }^{1} \mathrm{H}$ NMR $\left(500 \mathrm{MHz}, \mathrm{CDCl}_{3}\right) \square 4.62$ (dt, $J=$ 4.5, 8.6 Hz, 1H), 3.62 (s, 3H), 3.08 (s, 3H), $2.76(\mathrm{dd}, J=7.8,15.3 \mathrm{~Hz}, 1 \mathrm{H}), 2.64$ (dd, J = 4.8, 15.3 Hz, 1H), $1.95(\mathrm{~s}, 3 \mathrm{H}), 0.92(\mathrm{~d}, J=5.9 \mathrm{~Hz}, 3 \mathrm{H}), 0.83(\mathrm{~m}, 1 \mathrm{H}), 0.71(\mathrm{tt}, J=5.1,9.1 \mathrm{~Hz}, 1 \mathrm{H}), 0.38(\mathrm{dt}, J=4.8,8.6 \mathrm{~Hz}$, $1 \mathrm{H}), 0.21(\mathrm{dt}, J=5.1,8.6 \mathrm{~Hz}, 1 \mathrm{H}) ;{ }^{13} \mathrm{C} \mathrm{NMR}\left(125 \mathrm{MHz}, \mathrm{CDCl}_{3}\right) \square 171.2,170.3,74.4,61.3,37.3,32.2,23.7$, 21.2, 18.4, 12.1, 11.2; high resolution mass spectrum $(\mathrm{CI}+) \mathrm{m} / z 230.1405\left[(\mathrm{M})^{+}\right.$; calcd for $\mathrm{C}_{11} \mathrm{H}_{19} \mathrm{O}_{4} \mathrm{~N}$ : 230.1392].

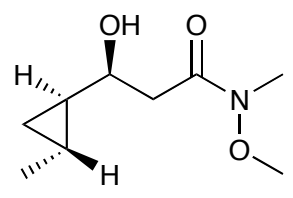

$(-)-11$

Anti-cyclopropyl carbinol (-)-11. To a $0{ }^{0} \mathrm{C}$ solution of acetate (-)-SI 1 (2.50 g, $\left.10.9 \mathrm{mmol}\right)$ in $\mathrm{MeOH}(285$ $\mathrm{mL}$ ) was added $\mathrm{K}_{2} \mathrm{CO}_{3}(1.66 \mathrm{~g}, 1.10 \mathrm{eq})$. After $17 \mathrm{~h}$ at $0{ }^{\circ} \mathrm{C}$, the reaction was quenched with saturated $\mathrm{NH}_{4} \mathrm{Cl}$ (2.0 mL) and concentrated. The resulting suspension was partitioned between $\mathrm{H}_{2} \mathrm{O}(50 \mathrm{~mL})$ and EtOAc (100 $\mathrm{mL})$, and the layers were separated. The aqueous layer was then extracted with EtOAc (100 mL, 3x), and the combined organic layers were dried over $\mathrm{MgSO}_{4}$ and concentrated. Flash chromatography $(2: 1=$ EtOAc:hex) afforded anti-cyclopropylcarbinol (-)-11 (1.62 g, 78\% yield) as a yellow oil. [ []$_{\mathrm{D}}{ }^{20}-80.6(c$ 1.44, $\mathrm{CHCl}_{3}$ ); IR (thin film, $\mathrm{CDCl}_{3}$ ) 3418 (br, w), 2946 (w), 1638 (m), 1445 (w), 1388 (w) cm ${ }^{-1} ;{ }^{1} \mathrm{H} \mathrm{NMR}$ $\left(500 \mathrm{MHz}, \mathrm{CDCl}_{3}\right) \square 3.66(\mathrm{~s}, 3 \mathrm{H}), 3.29(\mathrm{dt}, J=2.2,8.6 \mathrm{~Hz}, 1 \mathrm{H}), 3.15(\mathrm{~s}, 3 \mathrm{H}), 2.70(\mathrm{~d}, J=16.4 \mathrm{~Hz}, 1 \mathrm{H}), 2.58$ $(\mathrm{dd}, J=6.7,16.4 \mathrm{~Hz}, 1 \mathrm{H}), 1.02(\mathrm{~d}, J=6.7 \mathrm{~Hz}, 3 \mathrm{H}), 0.79(\mathrm{~m}, 1 \mathrm{H}), 0.63(\mathrm{~m}, 1 \mathrm{H}), 0.30(\mathrm{dt}, J=4.8,8.2 \mathrm{~Hz}$, 1H), $0.18(\mathrm{dt}, J=4.8,8.4 \mathrm{~Hz}, 1 \mathrm{H}) ;{ }^{13} \mathrm{C} \mathrm{NMR}\left(125 \mathrm{MHz}, \mathrm{CDCl}_{3}\right) \square 173.3,72.5,61.3,38.1,31.9,25.4,18.7$, 11.6, 10.5; high resolution mass spectrum $(\mathrm{CI}+) \mathrm{m} / \mathrm{z} \quad 170.1186\left[(\mathrm{M}-\mathrm{OH})^{+}\right.$; calcd for $\left.\mathrm{C}_{9} \mathrm{H}_{16} \mathrm{O}_{2} \mathrm{~N}: 170.1181\right]$. 


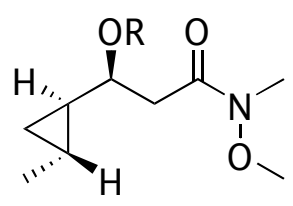

$(-)-S I 2$

$R=$ TIPS

Silyl ether (-)-SI 2. To a $0{ }^{\circ} \mathrm{C}$ solution of alcohol (-)-11 (1.58 g, $\left.8.5 \mathrm{mmol}\right)$ and 2,6-lutidine (1.97 mL, $\left.2.0 \mathrm{eq}\right)$ in $\mathrm{CH}_{2} \mathrm{Cl}_{2}(60 \mathrm{~mL})$ was added TIPSOTf (triisopropylsilyl-trifluoromethanesulfonate) (3.41 mL, $\left.1.50 \mathrm{eq}\right)$. After $1 \mathrm{~h}$, the reaction was quenched with saturated $\mathrm{NaHCO}_{3}(20 \mathrm{~mL})$ and the layers were separated. The aqueous layer was then extracted with $\mathrm{CH}_{2} \mathrm{Cl}_{2}(60 \mathrm{~mL}, 3 \mathrm{x})$, and the combined organic layers were washed with $1 \mathrm{M} \mathrm{HCl}$, saturated $\mathrm{NaHCO}_{3}$ and brine $\left(50 \mathrm{~mL}\right.$ each), dried over $\mathrm{MgSO}_{4}$ and concentrated. Flash

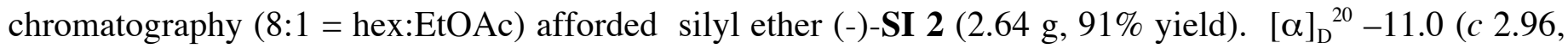
$\mathrm{CHCl}_{3}$ ); IR (thin film, $\left.\mathrm{CDCl}_{3}\right) 2946$ (br, m), 2866 (m), 1663 (m), 1383 (m) cm ${ }^{-1} ;{ }^{1} \mathrm{H} \mathrm{NMR} \mathrm{(500} \mathrm{MHz,} \mathrm{CDCl}{ }_{3}$ ) $\square 3.80(\mathrm{q}, J=6.7 \mathrm{~Hz}, 1 \mathrm{H}), 3.67(\mathrm{~s}, 3 \mathrm{H}), 3.13(\mathrm{~s}, 3 \mathrm{H}), 2.73(\mathrm{dd}, J=6.3,13.8 \mathrm{~Hz}, 1 \mathrm{H}), 2.61(\mathrm{dd}, J=6.3,14.5$ $\mathrm{Hz}, 1 \mathrm{H}), 1.06-1.01(\mathrm{~m}, 21 \mathrm{H}), 0.99(\mathrm{~d}, J=6.0 \mathrm{~Hz}, 3 \mathrm{H}), 0.73(\mathrm{~m}, 1 \mathrm{H}), 0.69(\mathrm{~m}, 1 \mathrm{H}), 0.35(\mathrm{dt}, J=4.8,8.2 \mathrm{~Hz}$, $1 \mathrm{H}), 0.14(\mathrm{dt}, J=4.8,8.6 \mathrm{~Hz}, 1 \mathrm{H}) ;{ }^{13} \mathrm{C} \mathrm{NMR}\left(125 \mathrm{MHz}, \mathrm{CDCl}_{3}\right) \square 172.5,73.1,61.4,41.2,32.1,27.1,18.7$, $18.3(6 \mathrm{C}), 12.8(3 \mathrm{C}), 11.9,10.5$; high resolution mass spectrum $(\mathrm{ES}+) \mathrm{m} / \mathrm{z} 366.2457\left[(\mathrm{M}+\mathrm{Na})^{+}\right.$; calcd for $\left.\mathrm{C}_{18} \mathrm{H}_{37} \mathrm{O}_{3} \mathrm{NSiNa}^{+}: 366.2440\right]$.

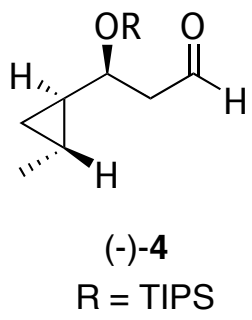

Aldehyde (-)-4. To a $-78{ }^{\circ} \mathrm{C}$ solution of Weinreb amide (-)-SI 2 (2.58 g, $\left.7.5 \mathrm{mmol}\right)$ in THF (45 mL) was slowly added DIBAL-H (9.03 mL, 1.0M in hexanes, $1.2 \mathrm{eq})$. After 5 minutes, the reaction was quenched with saturated Rochelle's salt solution $(30 \mathrm{~mL})$ and warmed to RT. The layers were then separated and the aqueous layer was extracted with $\mathrm{CH}_{2} \mathrm{Cl}_{2}(60 \mathrm{~mL}, 3 \mathrm{x})$. The combined organic layers were then dried over $\mathrm{MgSO}_{4}$ and concentrated. Flash chromatography (20:1 = hex:EtOAc) afforded aldehyde (-)-4 (1.89 g, $88 \%$ yield). [D] ${ }_{\mathrm{D}}^{20}-2.7$ ( $0.89, \mathrm{CHCl}_{3}$ ); IR (thin film, $\mathrm{CDCl}_{3}$ ) 2945 (s), 2867 (s), 1725 (s), 1464 (m), 1106 (s) cm ${ }^{1} ;{ }^{1} \mathrm{H}$ NMR (500 MHz, $\mathrm{CDCl}_{3}$ ) $\square 9.92$ (s, 1H), 3.73 (dt, $\left.J=5.6,7.5 \mathrm{~Hz}, 1 \mathrm{H}\right), 2.65$ (m, 2H), 1.09-1.06 (m, $21 \mathrm{H}), 1.04(\mathrm{~d}, J=6.0 \mathrm{~Hz}, 3 \mathrm{H}), 0.80(\mathrm{~m}, 1 \mathrm{H}), 0.76(\mathrm{~m}, 1 \mathrm{H}), 0.35(\mathrm{dt}, J=4.8,8.6 \mathrm{~Hz}, 1 \mathrm{H}), 0.26(\mathrm{dt}, J=5.1$, 8.2 Hz, 1H); ${ }^{13} \mathrm{C}$ NMR (125 MHz, $\left.\mathrm{CDCl}_{3}\right) \square 202.2$, 72.7, 52.3, 26.8, 18.6, 18.3 (6C), 12.8 (4C), 10.5; high resolution mass spectrum $(\mathrm{ES}+) \mathrm{m} / \mathrm{z} 307.2081$ [(M+Na) ${ }^{+}$calcd for $\mathrm{C}_{16} \mathrm{H}_{32} \mathrm{O}_{2} \mathrm{SiNa}^{+}$: 307.2069$]$. 


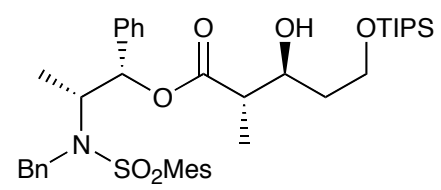

$(-)-13$

Anti-aldol product (-)-13. To a solution of chiral ester auxillary (-)-12 (47.0 g, $98.0 \mathrm{mmol})$ in $\mathrm{CH}_{2} \mathrm{Cl}_{2}(490$ $\mathrm{mL}$ ) was added $\mathrm{Et}_{3} \mathrm{~N}(33.1 \mathrm{~mL}, 2.40 \mathrm{eq})$. The solution was cooled to $-78{ }^{\circ} \mathrm{C}$ and treated with freshly prepared $\mathrm{cHex}_{2} \mathrm{BOTf}^{1}(139 \mathrm{~mL}, 1.53 \mathrm{M}$ solution, $2.20 \mathrm{eq})$ dropwise over 20 minutes. The enolate solution was then stirred for $3 \mathrm{~h}$ before the dropwise addition of aldehyde $7^{2}$. After $2 \mathrm{~h}$, the reaction mixture was allowed to warm to RT over $1 \mathrm{~h}$ and then quenched with $500 \mathrm{~mL} \mathrm{pH} 7$ buffer and $1 \mathrm{~L} \mathrm{MeOH}$. The resulting solution was then carefully treated with $30 \%$ aqueous $\mathrm{H}_{2} \mathrm{O}_{2}(190 \mathrm{~mL})$, allowed to stir overnight and concentrated. The semi-solid was partitioned between $500 \mathrm{~mL} \mathrm{H} \mathrm{H}_{2} \mathrm{O}$ and $1.1 \mathrm{~L} \mathrm{CH}_{2} \mathrm{Cl}_{2}$. The aqueous layer was extracted with $\mathrm{CH}_{2} \mathrm{Cl}_{2}(600 \mathrm{~mL}, 3 \mathrm{x})$, and the combined organic layers were washed with $\mathrm{H}_{2} \mathrm{O}(400 \mathrm{~mL}$, 3x), dried over $\mathrm{MgSO}_{4}$ and concentrated. Flash chromatography (9:1:1 then 7:1:1= hex:EtOAc: $\mathrm{CH}_{2} \mathrm{Cl}_{2}$ ). afforded alcohol (-)-13 (66.7 g, 96\% yield) as a single diastereomer. [0] ${ }_{\mathrm{D}}{ }^{20}-18.9$ (c 1.08, $\left.\mathrm{CHCl}_{3}\right)$; (thin film, $\left.\mathrm{CDCl}_{3}\right) 3488$ (br, m), 2941 (s), 2866 (s), 1742 (s), 1604 (m), 1456 (s), 1325 (s), 1153 (s) cm ${ }^{-1}$; ${ }^{1} \mathrm{H} \mathrm{NMR}$ $\left(500 \mathrm{MHz}, \mathrm{CDCl}_{3}\right) \square 7.36(\mathrm{~d}, J=7.0 \mathrm{~Hz}, 2 \mathrm{H}), 7.27-7.15(\mathrm{~m}, 6 \mathrm{H}), 6.90(\mathrm{~s}, 2 \mathrm{H}), 6.86(\mathrm{~d}, J=7.9 \mathrm{~Hz}, 2 \mathrm{H}), 5.82$ $(\mathrm{d}, J=4.1 \mathrm{~Hz}, 1 \mathrm{H}), 4.84(\mathrm{~d}, J=16.4 \mathrm{~Hz}, 1 \mathrm{H}), 4.64(\mathrm{~d}, J=16.4 \mathrm{~Hz}, 1 \mathrm{H}), 4.10$ (ddd, $J=4.0,6.7,14.9 \mathrm{~Hz}$, $1 \mathrm{H}), 4.02(\mathrm{~m}, 1 \mathrm{H}), 3.97(\mathrm{dt}, J=4.8,10.0 \mathrm{~Hz}, 1 \mathrm{H}), 3.89(\mathrm{dt}, J=6.0,10.4 \mathrm{~Hz}, 1 \mathrm{H}), 3.71(\mathrm{~d}, J=3.0 \mathrm{~Hz}, 1 \mathrm{H},-$ $\mathrm{OH}), 2.60(\mathrm{t}, J=7.1 \mathrm{~Hz}, 1 \mathrm{H}), 2.52(\mathrm{~s}, 6 \mathrm{H}), 2.30(\mathrm{~s}, 3 \mathrm{H}), 1.68(\mathrm{dt}, J=5.9,4.8 \mathrm{~Hz}, 2 \mathrm{H}), 1.18(\mathrm{~d}, J=7.1 \mathrm{~Hz}$, $3 \mathrm{H}), 1.12(\mathrm{~d}, J=6.7 \mathrm{~Hz}, 3 \mathrm{H}), 1.09-1.04(\mathrm{~m}, 21 \mathrm{H}) ;{ }^{13} \mathrm{C} \mathrm{NMR}\left(125 \mathrm{MHz}, \mathrm{CDCl}_{3}\right) \square 174.1,142.6,140.5$ (2C), 139.0, 138.7, 133.8, 132.3 (2C), 128.5 (2C), 128.4 (2C), 127.9, 127.8 (2C), 127.1, 126.1 (2C), 78.3, 73.5, 62.8, 57.0, 48.5, 46.1, 35.5, 23.1 (2C), 21.0, 18.1 (6C), 13.5, 13.4, 11.9 (3C); high resolution mass spectrum (ES+) $m / z, 732.3740\left[(\mathrm{M}+\mathrm{Na})^{+}\right.$; calcd for $\left.\mathrm{C}_{40} \mathrm{H}_{59} \mathrm{NO}_{6} \mathrm{SiSNa}^{+}: 732.3730\right]$.

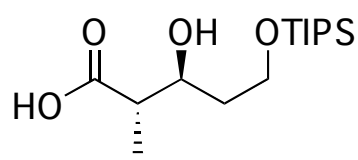

$(+)-5$

○-Hydroxy acid (+)-5. Chiral ester (-)-13 (1.12 g, $1.58 \mathrm{mmol})$ in THF $(10.5 \mathrm{~mL}) / \mathrm{H}_{2} \mathrm{O}(5.3 \mathrm{~mL})$ was treated with $\mathrm{LiOH}(0.189 \mathrm{~g}, 5.0 \mathrm{eq})$, and the resulting suspension was stirred for $72 \mathrm{~h}$ at RT. The reaction was then quenched with $60 \mathrm{~mL} \mathrm{H}_{2} \mathrm{O}$, and the mixture was acidified with $1 \mathrm{M} \mathrm{HCl}$ to $\mathrm{pH} 2$. The aqueous layer was then extracted with EtOAc $(100 \mathrm{~mL}, 3 \mathrm{x})$ and the combined organic layers were washed with saturated

${ }^{1}$ Abiko, A. Org. Syn. 2004, 10, 273.

${ }^{2}$ Rychnovsky, S. D.; Kopecky, D. J. J. Am. Chem. Soc. 2001, 34, 8420. 
$\mathrm{NH}_{4} \mathrm{Cl}(50 \mathrm{~mL})$, dried over $\mathrm{MgSO}_{4}$ and concentrated. Flash chromatography $(4: 1$ then 0:1 = hex:EtOAc)

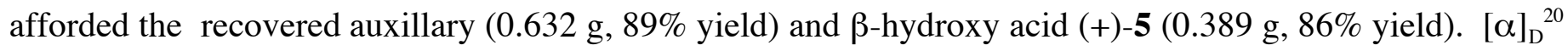
+3.0 (c 0.72, $\mathrm{CHCl}_{3}$ ); IR (thin film, $\mathrm{CDCl}_{3}$ ) 3446 (br, m), 2922 (br, m), 2866 (m), 1699 (br, s), 1472 (s) cm ${ }^{-1}$; ${ }^{1} \mathrm{H}$ NMR $\left(500 \mathrm{MHz}, \mathrm{CDCl}_{3}\right) \square 4.02(\mathrm{dt}, J=6.0,6.3 \mathrm{~Hz}, 1 \mathrm{H}), 3.96(\mathrm{dt}, J=4.5,10.0 \mathrm{~Hz}, 1 \mathrm{H}), 3.90(\mathrm{dt}, J=5.6$, $10.0 \mathrm{~Hz}, 1 \mathrm{H}), 2.59(\mathrm{dddd}, J=6.7,6.7,13.8,13.8 \mathrm{~Hz}, 1 \mathrm{H}), 1.73(\mathrm{~m}, 2 \mathrm{H}), 1.18(\mathrm{~d}, J=7.1 \mathrm{~Hz}, 3 \mathrm{H}), 1.06-1.00$ $(\mathrm{m}, 21 \mathrm{H}) ;{ }^{13} \mathrm{C}$ NMR $\left(125 \mathrm{MHz}, \mathrm{CDCl}_{3}\right) \square 180.0,72.9,62.7,45.4,35.6,17.9$ (6C), 13.3, 12.1 (3C); high resolution mass spectrum $(\mathrm{ES}+) \mathrm{m} / \mathrm{z}, 327.1958$ [(M+Na) ${ }^{+}$; calcd for $\mathrm{C}_{15} \mathrm{H}_{32} \mathrm{O}_{4} \mathrm{SiNa}^{+}$: 327.1968$]$.

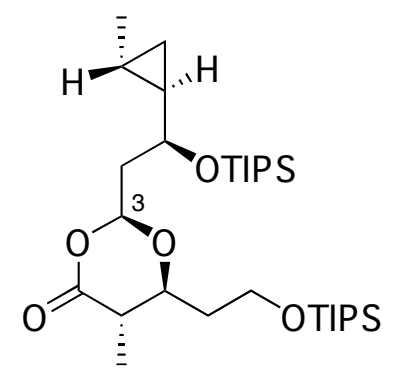

$(-)-16$

Dioxanone (-)-16. To a $35{ }^{\circ} \mathrm{C}$ solution of $\square$-hydroxy acid (+)-5 (0.206 g, $\left.0.678 \mathrm{mmol}\right)$ in $\mathrm{CH}_{2} \mathrm{Cl}_{2}(2.0 \mathrm{~mL})$ was added HMDS (0.502 mL, $4.0 \mathrm{eq})$, and the resulting solution was stirred for 4 days. Once complete, the reaction was concentrated, and the residual HMDS was removed under high vacuum (overnight) to afford the corresponding bis-TMS acid derivative $(0.304 \mathrm{~g}, 0.678 \mathrm{mmol})$, which was used without further purification. Aldehyde (-)-4 (0.249 g, $0.876 \mathrm{mmol}, 1.30 \mathrm{eq})$, the above TMS ester (0.304 g, $0.678 \mathrm{mmol})$ and DtBMP (2,6-di-tert-butyl-4-methyl pyridine) $(6.9 \mathrm{mg}, 0.05 \mathrm{eq})$ were azeotroped with benzene $(5 \mathrm{~mL}, 2 \mathrm{x})$ and then dissolved in $\mathrm{CH}_{2} \mathrm{Cl}_{2}(4.0 \mathrm{~mL})$. The reaction mixture was cooled to $-78{ }^{\circ} \mathrm{C}$, and TMSOTf (trimethylsilyltrifluoromethanesulfonate) (28 $\square \mathrm{L}, 0.22 \mathrm{eq})$ was added dropwise. After $1.5 \mathrm{~h}$, the reaction was quenched with pyridine $\left(28 \square \mathrm{L}\right.$ ), warmed to RT, and partitioned between $\mathrm{CH}_{2} \mathrm{Cl}_{2}(30 \mathrm{~mL})$ and saturated $\mathrm{NaHCO}_{3}(10$ $\mathrm{mL})$. The aqueous layer was then extracted with $\mathrm{CH}_{2} \mathrm{Cl}_{2}(30 \mathrm{~mL}, 3 \mathrm{x})$, and the combined organic layers were dried over $\mathrm{MgSO}_{4}$ and concentrated. Flash chromatography (10:1 = hex:EtOAc) using a short plug of silylated silica ${ }^{3}$ afforded dioxanone (-)-16 (0.351 g, 94\% yield over 2 steps) as an inseparable 7:1 mixture of C3 diastereomers. [ $\square]_{\mathrm{D}}{ }^{20}-39.7\left(c 0.87, \mathrm{C}_{6} \mathrm{H}_{6}\right.$ ); IR (thin film, $\left.\mathrm{C}_{6} \mathrm{D}_{6}\right) 2944$ (br, s), 2866 (s), 1751 (s), 1463 (s), 1248 (s), 1104 (s) cm ${ }^{-1}$; ${ }^{1} \mathrm{H}$ NMR $\left(500 \mathrm{MHz}, \mathrm{C}_{6} \mathrm{D}_{6}\right) \square 5.53(\mathrm{t}, J=6.0 \mathrm{~Hz}, 1 \mathrm{H}), 3.79$ (ddd, J= 4.1, 9.7, 9.7 Hz, $1 \mathrm{H}), 3.67(\mathrm{~m}, 1 \mathrm{H}), 3.62(\mathrm{t}, J=11.4 \mathrm{~Hz}, 1 \mathrm{H}), 3.55(\mathrm{dt}, J=6.4,8.1 \mathrm{~Hz}, 1 \mathrm{H}), 2.28-2.12(\mathrm{~m}, 3 \mathrm{H}), 1.60(\mathrm{~m}, 1 \mathrm{H})$, $1.39(\mathrm{ddd}, J=4.5,9.3,14.0 \mathrm{~Hz}, 1 \mathrm{H}), 1.20-1.12(\mathrm{~m}, 21 \mathrm{H}), 1.12-1.07(\mathrm{~m}, 21 \mathrm{H}), 1.07(\mathrm{~d}, J=7.0 \mathrm{~Hz}, 3 \mathrm{H}), 1.01$

\footnotetext{
${ }^{3}$ Silylated silica is prepared by adding TMSCl (trimethylsilyl chloride) $(38.4 \mathrm{~mL})$ to a suspension of regular silica $(430 \mathrm{~g})$ in $1.4 \mathrm{~L} \mathrm{CH}_{2} \mathrm{Cl}_{2}$. $\mathrm{The}$ resulting supenion is then cooled to $0{ }^{\circ} \mathrm{C}$ and $\mathrm{Et}_{3} \mathrm{~N}(98.4 \mathrm{~mL})$ is added dropwise over 20 minutes. Stirring is continued for $12 \mathrm{~h}$ at $\mathrm{RT}$ using a mechanical stirrer and then the silica was filtered and thoroughly washed with $\mathrm{CH}_{2} \mathrm{Cl}_{2}(200 \mathrm{~mL}, 10 \mathrm{x})$.
} 
$(\mathrm{d}, J=5.6 \mathrm{~Hz}, 3 \mathrm{H}), 0.75(\mathrm{~m}, 1 \mathrm{H}), 0.60(\mathrm{tt}, J=5.0,9.0 \mathrm{~Hz}, 1 \mathrm{H}), 0.30(\mathrm{dt}, J=4.8,8.6 \mathrm{~Hz}, 1 \mathrm{H}), 0.09(\mathrm{dt}, J=$ 5.2, 8.8 Hz, 1H); ${ }^{13} \mathrm{C}$ NMR (125 MHz, $\left.\mathrm{C}_{6} \mathrm{D}_{6}\right) \square 170.1,101.2,78.0,73.1,59.5,44.9,41.9,37.7,27.3,19.1$, 18.9 (6C), 18.6 (6C), 13.5 (3C), 13.1, 12.8, 12.6 (3C), 11.1; high resolution mass spectrum $(\mathrm{ES}+) \mathrm{m} / \mathrm{z}$ $593.4054\left[(\mathrm{M}+\mathrm{Na})^{+}\right.$; calcd for $\mathrm{C}_{31} \mathrm{H}_{62} \mathrm{O}_{5} \mathrm{Si}_{2} \mathrm{Na}^{+}$: 593.4033].

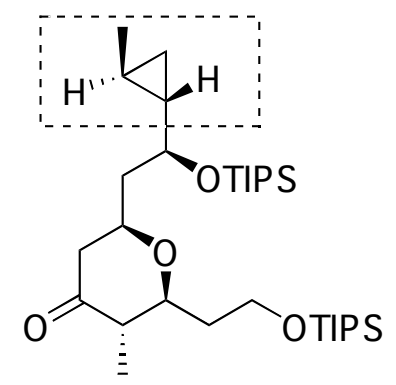

$(-)-15$

Pyranone (-)-15. To a RT solution of enol ether $14(0.173 \mathrm{~g}, 0.305 \mathrm{mmol})$ in $\mathrm{CH}_{2} \mathrm{Cl}_{2}(11.0 \mathrm{~mL})$ was quickly added $\mathrm{Me}_{2} \mathrm{AlCl}(0.335 \mathrm{~mL}, 1.0 \mathrm{M}$ solution in hexanes, $1.10 \mathrm{eq})$ in one portion and the reaction was immediately quenched after 1 second with saturated $\mathrm{NaHCO}_{3}(9.0 \mathrm{~mL})$. The aqueous layer was then extracted with $\mathrm{CH}_{2} \mathrm{Cl}_{2}(3 \mathrm{x}, 30 \mathrm{~mL})$, and the combined organic layers were washed with brine $(30 \mathrm{~mL})$, dried over $\mathrm{MgSO}_{4}$ and concentrated. Flash chromatography (25:1 = hex:EtOAc) afforded pyranone (-)-15 (0.103 $\mathrm{g}, 60 \%$ yield) as a light yellow oil. [ []$_{\mathrm{D}}{ }^{20}-15.1$ (c 2.00, $\mathrm{CHCl}_{3}$ ); IR (thin film, $\mathrm{CDCl}_{3}$ ) 2943 (br, s), 2866 (s), 1718 (s), 1463 (s), 1097 (s) cm ${ }^{-1} ;{ }^{1} \mathrm{H}$ NMR (500 MHz, $\mathrm{CDCl}_{3}$ ) $\square 3.86$ (ddd, $J=4.8,7.0,10.0 \mathrm{~Hz}, 1 \mathrm{H}$ ), 3.84$3.76(\mathrm{~m}, 2 \mathrm{H}), 3.54(\mathrm{dddd}, J=4.5,4.5,8.2,9.3 \mathrm{~Hz}, 1 \mathrm{H}), 3.37(\mathrm{dt}, J=2.6,10.0 \mathrm{~Hz}, 1 \mathrm{H}), 2.42(\mathrm{dd}, J=2.2$, $14.1 \mathrm{~Hz}, 1 \mathrm{H}), 2.38-2.28(\mathrm{~m}, 2 \mathrm{H}), 2.01(\mathrm{ddd}, J=4.1,9.7,13.8 \mathrm{~Hz}, 1 \mathrm{H}), 1.95(\mathrm{~m}, 1 \mathrm{H}), 1.74(\mathrm{dddd}, J=4.8,5.9$, 9.7, 14.0 Hz, 1H), 1.67 (ddd, $J=3.3,9.3,12.3 \mathrm{~Hz}, 1 \mathrm{H}), 1.08-1.04(\mathrm{~m}, 42 \mathrm{H}), 1.02$ (d, $J=6.7 \mathrm{~Hz}, 3 \mathrm{H}), 1.01$ $(\mathrm{d}, J=6.0 \mathrm{~Hz}, 3 \mathrm{H}), 0.65(\mathrm{~m}, 1 \mathrm{H}), 0.54(\mathrm{~m}, 1 \mathrm{H}), 0.45(\mathrm{dt}, J=4.5,8.6 \mathrm{~Hz}, 1 \mathrm{H}), 0.20(\mathrm{dt}, J=4.8,8.2 \mathrm{~Hz}, 1 \mathrm{H})$; ${ }^{13} \mathrm{C}$ NMR $\left(125 \mathrm{MHz}, \mathrm{CDCl}_{3}\right) \square 209.1,79.7,74.5,72.5,60.0,50.4,49.0,46.2,37.9,26.3,18.8,18.4$ (6C), 18.3 (6C), 13.0 (3C), 12.2 (3C), 11.4, 10.7, 9.5; high resolution mass spectrum (ES+) $\mathrm{m} / z \quad 591.4263$ $\left[(\mathrm{M}+\mathrm{Na})^{+}\right.$; calcd for $\mathrm{C}_{32} \mathrm{H}_{64} \mathrm{O}_{4} \mathrm{SiNa}^{+}:$591.4241]. 


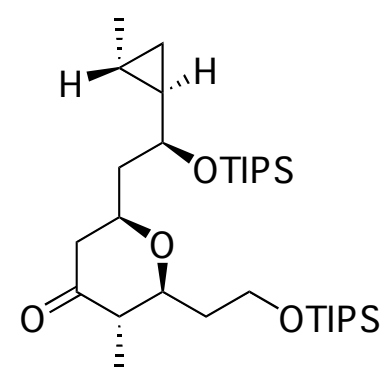

$(-)-18$

Pyranone (-)-18. Dioxanone (-)-16 (0.121 g, $0.213 \mathrm{mmol})$ in THF (1.0 mL) was treated with freshly prepared Petasis-Tebbe reagent ${ }^{4}(1.07 \mathrm{~mL}, 0.5 \mathrm{M}$ solution in THF, $2.50 \mathrm{eq})$ and ethyl trimethylacetate (4.9 $\square \mathrm{L}, 0.15 \mathrm{eq}$ ) at RT, and the resulting solution heated below reflux at $63{ }^{\circ} \mathrm{C}$ for $24 \mathrm{~h}$ in the absence of light. Upon completion, the reaction was cooled to RT, diluted with hexanes $(15 \mathrm{~mL})$, and filtered through a pad of celite. The concentrated crude mixture was then passed through a plug of silylated silica ${ }^{3}(20: 1=$ hex:EtOAc) to afford the crude enol ether which was used without further purification.

The above enol ether $(0.121 \mathrm{~g}, 0.213 \mathrm{mmol})$ in $\mathrm{CH}_{2} \mathrm{Cl}_{2}(5.5 \mathrm{~mL})$ was stirred with $4 \mathrm{~A} \mathrm{MS}(40 \mathrm{mg})$ for $0.5 \mathrm{~h}$ at RT. $\mathrm{Me}_{2} \mathrm{AlCl}(0.277 \mathrm{~mL}, 1.0 \mathrm{M}$ solution in hexanes, $1.30 \mathrm{eq})$ was then added quickly in one portion (gas evolution, venting required) and the reaction was immediately quenched after 1 second with saturated $\mathrm{NaHCO}_{3}(7.0 \mathrm{~mL})$. The aqueous layer was then extracted with $\mathrm{CH}_{2} \mathrm{Cl}_{2}(3 \mathrm{x}, 20 \mathrm{~mL})$, and the combined organic layers were washed with brine $(20 \mathrm{~mL})$, dried over $\mathrm{MgSO}_{4}$ and concentrated. Flash chromatography $(25: 1=\text { hex:EtOAc) afforded pyranone }(-)-18(79.0 \mathrm{mg}, 65 \% \text { yield, } 2 \text { steps }) \text { as a light yellow oil. [D] }]_{\mathrm{D}}^{20}$ -27.2 (c 2.30, $\mathrm{CHCl}_{3}$ ); IR (thin film, $\mathrm{CDCl}_{3}$ ) 2943 (br, s), 2866 (s), 1718 (s), 1463 (s), 1097 (br, s) cm ${ }^{-1} ;{ }^{1} \mathrm{H}$ $\operatorname{NMR}\left(500 \mathrm{MHz}, \mathrm{CDCl}_{3}\right) \square 3.85(\mathrm{~m}, 2 \mathrm{H}), 3.81(\mathrm{t}, J=8.2 \mathrm{~Hz}, 1 \mathrm{H}), 3.49(\mathrm{dt}, J=3.4,8.6 \mathrm{~Hz}, 1 \mathrm{H}), 3.36(\mathrm{dt}, J=$ 2.2, $9.7 \mathrm{~Hz}, 1 \mathrm{H}), 2.41(\mathrm{dd}, J=2.6,13.4 \mathrm{~Hz}, 1 \mathrm{H}), 2.34(\mathrm{~d}, J=11.9 \mathrm{~Hz}, 1 \mathrm{H}), 2.30$ (m, 1H), 1.99 (ddd, $J=4.1$, 9.3, $13.8 \mathrm{~Hz}, 1 \mathrm{H}), 1.94(\mathrm{br} \mathrm{d}, J=8.2 \mathrm{~Hz}, 1 \mathrm{H}), 1.70(\mathrm{~m}, 2 \mathrm{H}), 1.10-1.03(\mathrm{~m}, 42 \mathrm{H}), 1.02(\mathrm{~d}, J=5.9 \mathrm{~Hz}, 3 \mathrm{H})$, $1.01(\mathrm{~d}, J=6.3 \mathrm{~Hz}, 3 \mathrm{H}), 0.65(\mathrm{~m}, 1 \mathrm{H}), 0.57(\mathrm{~m}, 1 \mathrm{H}), 0.41(\mathrm{dt}, J=4.5,8.6 \mathrm{~Hz}, 1 \mathrm{H}), 0.19(\mathrm{dt}, J=4.8,8.6 \mathrm{~Hz}$, $1 \mathrm{H}) ;{ }^{13} \mathrm{C} \mathrm{NMR}\left(125 \mathrm{MHz}, \mathrm{CDCl}_{3}\right) \square 209.1,79.5,74.6,73.0,59.9,50.3,49.0,46.2,37.9,26.1,18.9,18.4$ (6C), 18.2 (6C), 12.9 (3C), 12.2 (3C), 11.0, 10.9, 9.5; high resolution mass spectrum (ES+) $\mathrm{m} / z \quad 591.4267$ $\left[(\mathrm{M}+\mathrm{Na})^{+}\right.$; calcd for $\mathrm{C}_{32} \mathrm{H}_{64} \mathrm{O}_{4} \mathrm{SiNa}^{+}$: 591.4241].

\footnotetext{
${ }^{4}$ Petasis-Tebbe reagent was prepared by treating $\mathrm{Cp}_{2} \mathrm{TiCl}_{2}(70.0 \mathrm{mmol}, 17.4 \mathrm{~g})$ in $\mathrm{Et}_{2} \mathrm{O}(250 \mathrm{~mL})$ with $\mathrm{MeLi}\left(3 \mathrm{eq}, 131 \mathrm{~mL}, 1.6 \mathrm{M}\right.$ in $\left.\mathrm{Et}_{2} \mathrm{O}\right)$ at $0{ }^{0} \mathrm{C}$ in a tared flask. After stirring for $2 \mathrm{~h}$, the reaction was quenched with saturated $\mathrm{NaHCO}_{3}(100 \mathrm{~mL})$ and stirred for 10 minutes before the layers were separated. The aqueous layer was then extracted with $\mathrm{Et}_{2} \mathrm{O}(200 \mathrm{~mL}, 3 \mathrm{x})$ and the combined organic layers were washed with saturated $\mathrm{NaHCO}_{3}(100 \mathrm{~mL})$. The organic layers were dried over $\mathrm{MgSO}_{4}$ and concentrated (water bath $<30{ }^{\circ} \mathrm{C}$; do not concentrate to dryness). After the solvent was almost fully removed, the flask was quickly weighed (14.5 g, quant.) and then covered with aluminum foil. The semi-solid was dissolved in THF to give $0.5 \mathrm{M}$ solution of the Petasis-Tebbe reagent. The stock solution was kept under argon at $0{ }^{\circ} \mathrm{C}$ in the absence of light..
} 


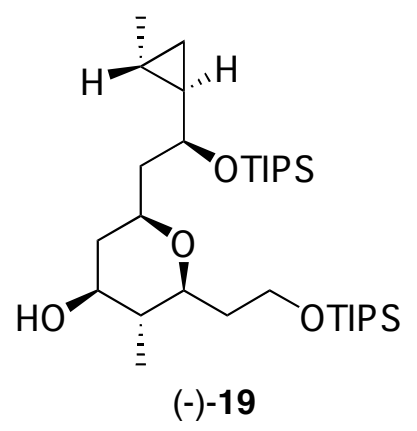

Alcohol (-)-19. To a $-10{ }^{\circ} \mathrm{C}$ solution of pyranone (-)-18 (0.105 g, $\left.0.184 \mathrm{mmol}\right)$ in EtOH (4.0 mL) was added $\mathrm{NaBH}_{4}(13.9 \mathrm{mg}, 2.0 \mathrm{eq})$, and the resulting solution was stirred for 30 minutes. The reaction was quenched with saturated $\mathrm{NH}_{4} \mathrm{Cl}(8.0 \mathrm{~mL})$, then brine $(15 \mathrm{~mL})$ and diluted with $\mathrm{CH}_{2} \mathrm{Cl}_{2}(40 \mathrm{~mL})$. The layers were then separated, and the aqueous layer was extracted with $\mathrm{CH}_{2} \mathrm{Cl}_{2}(50 \mathrm{~mL}, 3 \mathrm{x})$. The combined organic layers were then washed with brine $(30 \mathrm{~mL})$, dried over $\mathrm{MgSO}_{4}$ and concentrated. Flash chromatography (15:1 to 10:1 = hex:EtOAc) afforded alcohol (-)-19 (76.0 mg, 76\% yield). [D] ${ }_{\mathrm{D}}{ }^{20}-12.1$ (c 0.85, $\mathrm{CHCl}_{3}$ ); IR (thin film, $\left.\mathrm{CDCl}_{3}\right) 3347$ (br, w), 2943 (s), 2866 (s), 1463 (m), 1098 (m) cm ${ }^{-1} ;{ }^{1} \mathrm{H}$ NMR (500 MHz, CDCl ${ }_{3}$ ) $\square 3.80$ (ddd, $J$ $=4.5,7.8,9.7 \mathrm{~Hz}, 1 \mathrm{H}), 3.75(\mathrm{dt}, J=7.4,9.3 \mathrm{~Hz}, 1 \mathrm{H}), 3.58(\mathrm{t}, J=10.0 \mathrm{~Hz}, 1 \mathrm{H}), 3.45(\mathrm{ddd}, J=4.5,8.6,12.3$ $\mathrm{Hz}, 1 \mathrm{H}), 3.33(\mathrm{dt}, J=4.5,10.8 \mathrm{~Hz}, 1 \mathrm{H}), 3.06(\mathrm{dt}, J=2.2,10.1 \mathrm{~Hz}, 1 \mathrm{H}), 1.94(\mathrm{ddd}, J=1.9,4.8,12.8 \mathrm{~Hz}, 1 \mathrm{H})$, $1.88(\mathrm{~m}, 2 \mathrm{H}), 1.59(\mathrm{~m}, 2 \mathrm{H}), 1.26(\mathrm{q}, J=11.5 \mathrm{~Hz}, 1 \mathrm{H}), 1.21(\mathrm{~m}, 1 \mathrm{H}), 1.09-1.05(\mathrm{~m}, 42 \mathrm{H}), 1.03(\mathrm{~d}, J=5.9 \mathrm{~Hz}$, $3 \mathrm{H}), 0.99(\mathrm{~d}, J=6.3 \mathrm{~Hz}, 3 \mathrm{H}), 0.65(\mathrm{~m}, 1 \mathrm{H}), 0.58(\mathrm{tt}, J=5.1,8.8 \mathrm{~Hz}, 1 \mathrm{H}), 0.42(\mathrm{dt}, J=5.2,8.6 \mathrm{~Hz}, 1 \mathrm{H}), 0.19$ $(\mathrm{dt}, J=4.8,8.6 \mathrm{~Hz}, 1 \mathrm{H}) ;{ }^{13} \mathrm{C} \mathrm{NMR}\left(125 \mathrm{MHz}, \mathrm{CDCl}_{3}\right) \square 77.7,74.1,73.2,72.3,60.6,45.8,44.2,42.2,36.8$, 26.2, 19.0, 18.5 (6C), 18.3 (6C), 13.2, 13.0 (3C), 12.2 (3C), 11.0, 10.8; high resolution mass spectrum (ES+) $m / z$ 593.4412 [(M+Na) $)^{+}$; calcd for $\left.\mathrm{C}_{32} \mathrm{H}_{66} \mathrm{O}_{4} \mathrm{Si}_{2} \mathrm{Na}^{+}: 593.4397\right]$.

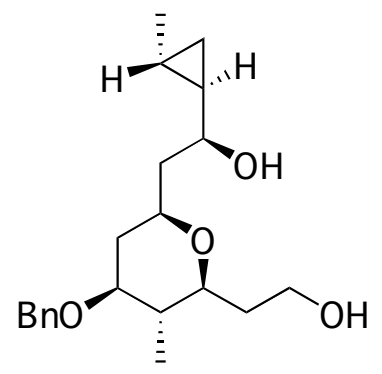

(+)-20

Diol (+)-20. Alcohol (-)-19 (68.1 mg, $0.119 \mathrm{mmol})$ in DMF (0.60 mL) was treated with NaH $(9.6 \mathrm{mg}, 60 \%$ wt. in oil, 2.0 eq) at RT for 30 minutes before adding $\mathrm{BnBr}(29 \square \mathrm{L}, 2.0 \mathrm{eq})$ and TBAI (tetrabutylammonium iodide) $(8.8 \mathrm{mg}, 0.20 \mathrm{eq})$. The resulting solution was then stirred overnight, and diluted with $\mathrm{H}_{2} \mathrm{O}(4.0 \mathrm{~mL})$ and $\mathrm{Et}_{2} \mathrm{O}(10 \mathrm{~mL})$. The aqueous layer was then extracted with $\mathrm{Et}_{2} \mathrm{O}(10 \mathrm{~mL}, 4 \mathrm{x})$, and the combined organic layers were washed with saturated $\mathrm{NH}_{4} \mathrm{Cl}$ and brine $\left(10 \mathrm{~mL}\right.$ each), dried over $\mathrm{MgSO}_{4}$ and concentrated to afford the corresponding benzyl ether, which was used without further purification. 
The above benzyl ether in $\mathrm{EtOH}(4.10 \mathrm{~mL})$ was treated with $1 \% \mathrm{HCl}(1.25 \mathrm{~mL})$, and the resulting solution was stirred for $24 \mathrm{~h}$ at RT. Once complete, the reaction was neutralized with saturated $\mathrm{NaHCO}_{3}(4.0 \mathrm{~mL})$ and the aqueous phase was extracted with EtOAc $(10 \mathrm{~mL}, 4 \mathrm{x})$. The combined organic layers were then dried over $\mathrm{MgSO}_{4}$ and concentrated. Flash chromatography (5:1 = $\mathrm{Et}_{2} \mathrm{O}$ :hex, then EtOAc) afforded diol (+)-20 (34.7 mg, 84\% over 2 steps). [ [ $]_{\mathrm{D}}{ }^{20}+26.3$ (c 1.92, $\mathrm{CHCl}_{3}$ ); IR (thin film, $\left.\mathrm{CDCl}_{3}\right) 3410(\mathrm{~m}), 2944$ (m), 1454 (m), $1072(\mathrm{~s}) \mathrm{cm}^{-1} ;{ }^{1} \mathrm{H}$ NMR (500 MHz, $\left.\mathrm{CDCl}_{3}\right) \square 7.38-7.27(\mathrm{~m}, 5 \mathrm{H}), 4.65(\mathrm{~d}, J=11.5 \mathrm{~Hz}, 1 \mathrm{H}), 4.43(\mathrm{~d}, J=$ $11.5 \mathrm{~Hz}, 1 \mathrm{H}), 3.75(\mathrm{dt}, J=4.8,7.0 \mathrm{~Hz}, 2 \mathrm{H}), 3.57$ (t, $J=10.0 \mathrm{~Hz}, 1 \mathrm{H}), 3.23$ (dt, $J=2.6,9.7 \mathrm{~Hz}, 1 \mathrm{H}), 3.12(\mathrm{~m}$, 2H), $2.12(\mathrm{ddd}, J=1.5,4.1,12.5 \mathrm{~Hz}, 1 \mathrm{H}), 1.93(\mathrm{~m}, 1 \mathrm{H}), 1.85(\mathrm{dt}, J=9.3,14.5 \mathrm{~Hz}, 1 \mathrm{H}), 1.70(\mathrm{dt}, J=3.4$, $14.5 \mathrm{~Hz}, 1 \mathrm{H}), 1.66(\mathrm{~m}, 1 \mathrm{H}), 1.49(\mathrm{~m}, 1 \mathrm{H}), 1.34(\mathrm{q}, J=11.2 \mathrm{~Hz}, 1 \mathrm{H}), 1.06(\mathrm{~d}, J=5.9 \mathrm{~Hz}, 3 \mathrm{H}), 0.99$ (d, $J=6.3$ $\mathrm{Hz}, 3 \mathrm{H}), 0.76(\mathrm{~m}, 1 \mathrm{H}), 0.62(\mathrm{tt}, J=5.0,9.3 \mathrm{~Hz}, 1 \mathrm{H}), 0.33(\mathrm{dt}, J=4.5,8.6 \mathrm{~Hz}, 1 \mathrm{H}), 0.23(\mathrm{dt}, J=4.5,8.2 \mathrm{~Hz}$, $1 \mathrm{H}) ;{ }^{13} \mathrm{C}$ NMR (125 MHz, $\mathrm{CDCl}_{3}$ ) $\square 138.7,128.6$ (2C), 127.9 (2C), 127.8, 81.1, 80.1, 76.0, 75.7, 70.7, 60.8, 42.9, 42.0, 37.6, 35.2, 26.8, 18.8, 16.7, 13.4, 11.3; high resolution mass spectrum (ES+) $\mathrm{m} / z \quad 371.2185$ $\left[(\mathrm{M}+\mathrm{Na})^{+}\right.$; calcd for $\mathrm{C}_{21} \mathrm{H}_{32} \mathrm{O}_{4} \mathrm{Na}^{+}$: 371.2198].

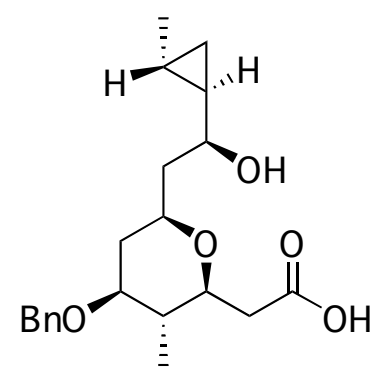

$(+)-3$

Monomer (+)-3. To a solution of diol (+)-20 (34.6 mg, $99.3 \square \mathrm{mol})$ and TEMPO (2,2,6,6tetramethylpiperidine-1-oxyl) $(0.776 \mathrm{mg}, 0.05 \mathrm{eq})$ in $\mathrm{CH}_{2} \mathrm{Cl}_{2}(0.44 \mathrm{~mL})$ was added saturated $\mathrm{NaHCO}_{3}(0.24$ $\mathrm{mL}) . \mathrm{KBr}(1.2 \mathrm{mg}, 0.10 \mathrm{eq})$ and TBAC (tetrabutylammonium chloride) (1.93 $\mathrm{mg}, 0.07 \mathrm{eq})$ were then added as aqueous stock solutions and the biphasic reaction mixture cooled to $0{ }^{\circ} \mathrm{C}$. To this vigorously stirring solution was then added a mixture of bleach $(0.379 \mathrm{~mL}, 0.72 \mathrm{M}$ solution, $2.75 \mathrm{eq})$, saturated $\mathrm{NaHCO}_{3}(0.14$ $\mathrm{mL})$ and brine $(0.26 \mathrm{~mL})$ dropwise over 45 minutes via syringe pump. Once the addition was complete, the reaction was stirred for an additional 30 minutes, and then diluted with $\mathrm{H}_{2} \mathrm{O}(1.6 \mathrm{~mL})$ and $\mathrm{Et}_{2} \mathrm{O}(4.0 \mathrm{~mL})$. The entire mixture was then acidified to $\mathrm{pH} 3$ with $10 \%$ citric acid, and the aqueous phase was extracted with EtOAc $(10 \mathrm{~mL}, 5 \mathrm{x})$. The combined organic layers were then washed with brine $(5.0 \mathrm{~mL})$, dried over $\mathrm{MgSO}_{4}$ and concentrated. Flash chromatography $\left(1: 1=\mathrm{Et}_{2} \mathrm{O}:\right.$ hex, then 1:1 $\left.=\mathrm{Et}_{2} \mathrm{O}: \mathrm{hex} \mathrm{w} / 0.5 \% \mathrm{AcOH}\right)$ afforded monomer (+)-3 (29.1 mg, 81\% yield). [ [ $]_{\mathrm{D}}{ }^{20}+13.5$ (c 1.22, $\mathrm{CHCl}_{3}$ ); IR (thin film, $\left.\mathrm{CDCl}_{3}\right) 3459$ (br, w), 2942 (m), 1727 (s), 1082 (s) $\mathrm{cm}^{-1} ;{ }^{1} \mathrm{H}$ NMR (500 MHz, $\mathrm{CDCl}_{3}$ ) $\square 7.38-7.28(\mathrm{~m}, 5 \mathrm{H}), 4.65(\mathrm{~d}, J=11.2 \mathrm{~Hz}, 1 \mathrm{H})$, $4.44(\mathrm{~d}, J=11.2 \mathrm{~Hz}, 1 \mathrm{H}), 3.60(\mathrm{t}, J=10.0 \mathrm{~Hz}, 1 \mathrm{H}), 3.52(\mathrm{t}, J=10.4 \mathrm{~Hz}, 1 \mathrm{H}), 3.18(\mathrm{~m}, 2 \mathrm{H}), 2.66(\mathrm{dd}, J=2.2$, $15.3 \mathrm{~Hz}, 1 \mathrm{H}), 2.40(\mathrm{dd}, J=4.5,10.8 \mathrm{~Hz}, 1 \mathrm{H}), 2.09(\mathrm{dd}, J=4.1,12.3 \mathrm{~Hz}, 1 \mathrm{H}), 1.83(\mathrm{dt}, J=9.7,14.9 \mathrm{~Hz}, 1 \mathrm{H})$, 
$1.70(\mathrm{~d}, J=14.9 \mathrm{~Hz}, 1 \mathrm{H}), 1.46(\mathrm{~m}, 1 \mathrm{H}), 1.37(\mathrm{q}, J=11.9 \mathrm{~Hz}, 1 \mathrm{H}), 1.01(\mathrm{~d}, J=5.9 \mathrm{~Hz}, 3 \mathrm{H}), 0.99(\mathrm{~d}, J=6.3$ $\mathrm{Hz}, 3 \mathrm{H}), 0.78(\mathrm{~m}, 1 \mathrm{H}), 0.60(\mathrm{tt}, J=5.0,8.7 \mathrm{~Hz}, 1 \mathrm{H}), 0.26(\mathrm{dt}, J=4.5,8.6 \mathrm{~Hz}, 1 \mathrm{H}), 0.18(\mathrm{dt}, J=4.8,8.6 \mathrm{~Hz}$, $1 \mathrm{H}) ;{ }^{13} \mathrm{C} \mathrm{NMR}\left(125 \mathrm{MHz}, \mathrm{CDCl}_{3}\right) \square 173.6,138.6,128.6$ (2C), 127.9 (2C), 127.9, 79.7, 78.6, 77.6, 76.8, 70.8, 42.1, 41.8, 38.8, 37.6, 25.9, 18.7, 13.3, 12.0, 10.4; high resolution mass spectrum (ES+) $\mathrm{m} / z \quad 385.2003$ $\left[(\mathrm{M}+\mathrm{Na})^{+}\right.$; calcd for $\mathrm{C}_{21} \mathrm{H}_{30} \mathrm{O}_{5} \mathrm{Na}^{+}$: 385.1991$]$.

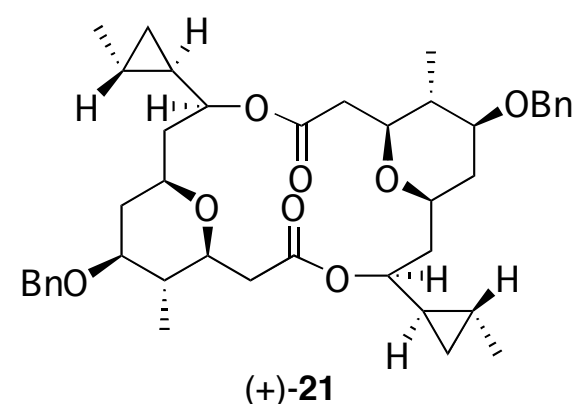

\section{Diolide (+)-21.}

Corey-Nicolaou method. Hydroxyacid (+)-3 $(28.0 \mathrm{mg}, 77.3 \square \mathrm{mol})$ in $\mathrm{CH}_{2} \mathrm{Cl}_{2}(1.0 \mathrm{~mL})$ was treated with $\mathrm{Py}_{2} \mathrm{~S}_{2}(23.0 \mathrm{mg}, 1.35 \mathrm{eq})$ and $\mathrm{PPh}_{3}(27.4 \mathrm{mg}, 1.35 \mathrm{eq})$ and stirred for $12 \mathrm{~h}$ at RT. The reaction mixture was then concentrated and passed through a short plug of silica $\left(6: 1=\mathrm{Et}_{2} \mathrm{O}: \mathrm{hex}\right)$ to afford the intermediate thioester which was used without further purification.

The thioester (azeotroped from benzene, $2 \mathrm{x}$ ) in toluene $(4.0 \mathrm{~mL}$ ) was then added via syringe pump to toluene $(22.0 \mathrm{~mL})$ at reflux $\left(125^{\circ} \mathrm{C}\right)$ over $15 \mathrm{~h}$. Once the first addition was complete, the original flask was rinsed with toluene $(1.0 \mathrm{~mL})$, and added to the reaction over 30 minutes. The reaction was then heated at reflux for an additional $24 \mathrm{~h}$, cooled to RT and concentrated. Flash chromatography $(6: 1=$ hex:EtOAc) afforded diolide (+)-21 (8.2 mg, 30\% yield).

Yamaguchi method. To a RT solution of monomer (+)-3 (56.6 mg, $0.156 \mathrm{mmol})$ in THF (1.0 mL) was added $\mathrm{Et}_{3} \mathrm{~N}(31 \square \mathrm{L}, 1.4 \mathrm{eq}$ ) and 2,4,6-trichlorobenzoyl chloride (30 $\square \mathrm{L}, 1.2 \mathrm{eq}$ ), and the resulting solution was stirred for $2 \mathrm{~h}$. The suspension was then filtered, the filtrate diluted with toluene $(8.0 \mathrm{~mL})$, and then added dropwise to a solution of DMAP (4-dimethylaminopyridine) (95.5 mg, $5.0 \mathrm{eq})$ in toluene (33.0 mL) at reflux $\left(125^{\circ} \mathrm{C}\right)$ over $4 \mathrm{~h}$. The original flask was rinsed with toluene $(1.0 \mathrm{~mL})$, and the solution added to the reaction mixture over 30 minutes. After an additional $2 \mathrm{~h}$ at reflux, the reaction was cooled to RT and concentrated. The crude product was partitioned between $\mathrm{H}_{2} \mathrm{O}(15 \mathrm{~mL})$ and EtOAc $(30 \mathrm{~mL})$, and the aqueous layer further extracted with EtOAc $(30 \mathrm{~mL}, 3 \mathrm{x})$. The combined organic layers were then washed with brine $(20 \mathrm{~mL})$, dried and concentrated. Flash chromatography $(6: 1=$ hex:EtOAc) afforded diolide $(+)$ 21 (37.1 mg, 66\% yield). [ []$_{\mathrm{D}}{ }^{20}+1.7$ (c 1.27, $\mathrm{CHCl}_{3}$ ); IR (thin film, $\left.\mathrm{CDCl}_{3}\right) 2949(\mathrm{~m}), 2867(\mathrm{~m}), 1734(\mathrm{~s})$, $1181(\mathrm{~m}), 1092$ (s) $\mathrm{cm}^{-1}$; ${ }^{1} \mathrm{H}$ NMR $\left(500 \mathrm{MHz}, \mathrm{CDCl}_{3}\right) \square 7.38-7.27(\mathrm{~m}, 10 \mathrm{H}), 4.65(\mathrm{~d}, J=11.5 \mathrm{~Hz}, 2 \mathrm{H}), 4.43$ $(\mathrm{d}, J=11.5 \mathrm{~Hz}, 2 \mathrm{H}), 4.42(\mathrm{t}, J=7.0 \mathrm{~Hz}, 2 \mathrm{H}), 3.44(\mathrm{~m}, 4 \mathrm{H}), 3.14(\mathrm{dt}, J=4.5,10.4 \mathrm{~Hz}, 2 \mathrm{H}), 2.56(\mathrm{dd}, J=3.7$, $17.5 \mathrm{~Hz}, 2 \mathrm{H}), 2.44(\mathrm{dd}, J=6.7,17.5 \mathrm{~Hz}, 2 \mathrm{H}), 2.10(\mathrm{dd}, J=4.8,11.9 \mathrm{~Hz}, 2 \mathrm{H}), 1.95(\mathrm{dt}, J=8.9,14.9 \mathrm{~Hz}, 2 \mathrm{H})$, 
$1.71(\mathrm{~d}, J=14.9 \mathrm{~Hz}, 2 \mathrm{H}), 1.40(\mathrm{~m}, 2 \mathrm{H}), 1.25(\mathrm{q}, J=11.9 \mathrm{~Hz}, 2 \mathrm{H}), 0.99(\mathrm{~d}, J=6.0 \mathrm{~Hz}, 6 \mathrm{H}), 0.94(\mathrm{~d}, J=6.7$ $\mathrm{Hz}, 6 \mathrm{H}), 0.83(\mathrm{~m}, 2 \mathrm{H}), 0.74(\mathrm{tt}, J=4.8,8.8 \mathrm{~Hz}, 2 \mathrm{H}), 0.37(\mathrm{dt}, J=4.8,8.5 \mathrm{~Hz}, 2 \mathrm{H}), 0.25(\mathrm{dt}, J=5.1,8.2 \mathrm{~Hz}$, 2H); ${ }^{13} \mathrm{C}$ NMR (125 MHz, $\mathrm{CDCl}_{3}$ ) $\square 171.4$ (2C), 138.8 (2C), 128.6 (4C), 128.0 (4C), 127.8 (2C), 80.4 (2C), 77.6 (2C), 77.0 (2C), 75.0 (2C), 70.9 (2C), 42.4 (2C), 41.8 (2C), 39.5 (2C), 37.8 (2C), 24.9 (2C), $18.7(2 \mathrm{C})$, $13.2(2 \mathrm{C}), 12.1(2 \mathrm{C}), 11.1(2 \mathrm{C})$; high resolution mass spectrum $(\mathrm{ES}+) \mathrm{m} / z \quad 711.3847\left[(\mathrm{M}+\mathrm{Na})^{+}\right.$; calcd for $\mathrm{C}_{42} \mathrm{H}_{56} \mathrm{O}_{8} \mathrm{Na}^{+}$: 711.3873].

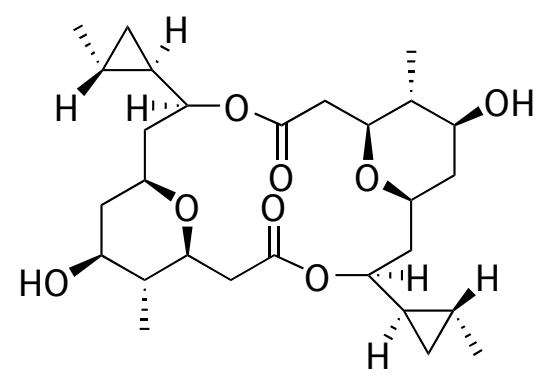

$(-)-2$

Aglycon (-)-2. To a solution of diolide (+)-21 (7.5 mg, $11 \square \mathrm{mol})$ in EtOH (500 $\square \mathrm{L}$ ) was added 10\% wt. Pd/C $\left(2.0 \mathrm{mg}\right.$, then additional $2.0 \mathrm{mg}$ after $8 \mathrm{~h}$ ), and the reaction flask was purged with $\mathrm{H}_{2}(3 \mathrm{x})$. The reaction was then allowed to stir for $12 \mathrm{~h}$ under $\mathrm{H}_{2}(1 \mathrm{~atm})$, then was filtered through a pad of Celite, washed with $\mathrm{MeOH}$ and concentrated. Flash chromatography $(1: 1=$ hex:EtOAc) afforded aglycon (-)-2 (3.5 mg, 64\% yield). $[\square]_{\mathrm{D}}{ }^{20}-40.2$ (c 0.16, $\mathrm{CHCl}_{3}$ ); IR (thin film, $\mathrm{CDCl}_{3}$ ) 3430 (br, m), $2951(\mathrm{~m}), 1732(\mathrm{~s}), 1175(\mathrm{~m}), 1039(\mathrm{~m}) \mathrm{cm}^{-}$ ${ }^{1} ;{ }^{1} \mathrm{H}$ NMR $\left(500 \mathrm{MHz}, \mathrm{CDCl}_{3}\right) \square 4.43$ (d, $\left.J=9.0 \mathrm{~Hz}, 2 \mathrm{H}\right), 3.49$ (br t, $\left.J=9.0 \mathrm{~Hz}, 2 \mathrm{H}\right), 3.44$ (ddd, $J=3.5,6.5$, $10.0 \mathrm{~Hz}, 2 \mathrm{H}), 3.37(\mathrm{td}, J=4.5,10.0 \mathrm{~Hz}, 2 \mathrm{H}), 2.55(\mathrm{dd}, J=3.7,17.5 \mathrm{~Hz}, 2 \mathrm{H}), 2.43(\mathrm{dd}, J=6.5,17.5 \mathrm{~Hz}, 2 \mathrm{H})$, 1.95-1.91 (m, 4H), $1.70(\mathrm{dq}, J=3.5,15.0 \mathrm{~Hz}, 2 \mathrm{H}), 1.46(\mathrm{~d}, J=4.0 \mathrm{~Hz}, 2 \mathrm{H},-\mathrm{OH}), 1.32-1.20(\mathrm{~m}, 4 \mathrm{H}), 0.99(\mathrm{~d}$, $J=6.0 \mathrm{~Hz}, 6 \mathrm{H}), 0.96(\mathrm{~d}, J=6.5 \mathrm{~Hz}, 6 \mathrm{H}), 0.83(\mathrm{~m}, 2 \mathrm{H}), 0.74(\mathrm{tt}, J=5.0,9.0 \mathrm{~Hz}, 2 \mathrm{H}), 0.37(\mathrm{dt}, J=5.0,8.5$ $\mathrm{Hz}, 2 \mathrm{H}), 0.25$ (dt, $J=5.0,8.5 \mathrm{~Hz}, 2 \mathrm{H}) ;{ }^{13} \mathrm{C} \mathrm{NMR}\left(125 \mathrm{MHz}, \mathrm{CDCl}_{3}\right.$ ) $\square 171.3$ (2C), 77.1 (2C), 77.1 (2C), 75.0 (2C), 73.5 (2C), 44.3 (2C), 41.7 (2C), 41.6 (2C), 39.3 (2C), 24.9 (2C), $18.7(2 \mathrm{C}), 12.9$ (2C), $12.1(2 \mathrm{C}), 11.1$ (2C); high resolution mass spectrum $(\mathrm{ES}+) \mathrm{m} / z$. 531.2925 [(M+Na) ${ }^{+}$; calcd for $\mathrm{C}_{28} \mathrm{H}_{44} \mathrm{O}_{8} \mathrm{Na}^{+}$: 531.2934]. 


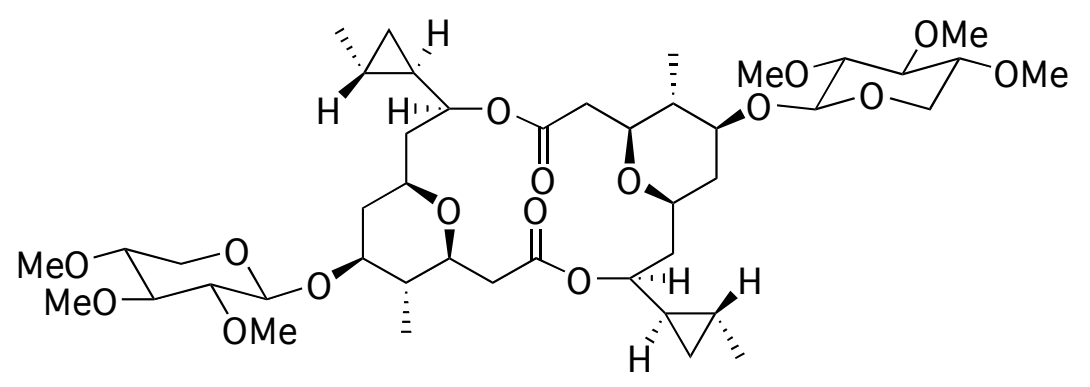

(-)-Clavosolide A

(-)-Clavosolide A (1). A solution of aglycon (-)-2 (8.0 mg, $15 \square \mathrm{mol})$ and trichloroacetimidate $22^{5}$ (78 $\square \mathrm{L}$, 0.5M solution in $\left.\mathrm{CH}_{2} \mathrm{Cl}_{2}, 2.5 \mathrm{eq}\right)$ in $\mathrm{CH}_{2} \mathrm{Cl}_{2}(400 \square \mathrm{L}$ ) was stirred with 4A MS (12 mg) for 30 minutes at RT. The solution was then cooled to $-20{ }^{\circ} \mathrm{C}$, and charged with TMSOTf (trimethylsilyltrifluoromethanesulfonate) (2 $\square \mathrm{L}, 1.0 \mathrm{M}$ solution in $\left.\mathrm{CH}_{2} \mathrm{Cl}_{2}, 0.13 \mathrm{eq}\right)$. The reaction was then allowed to slowly warm to RT over $1 \mathrm{~h}$, was quenched with $\mathrm{Et}_{3} \mathrm{~N}$ (1 drop) and concentrated. Flash chromatography (2:1 $=\mathrm{Et}_{2} \mathrm{O}$ :hex) afforded (-)-clavosolide $\mathrm{A}(\mathbf{1})\left(1.6 \mathrm{mg}, 12 \%\right.$ yield) as a white solid. [ []$_{\mathrm{D}}{ }^{20}-42.5$ (c 0.10, $\mathrm{CHCl}_{3}$ ); IR (thin film, $\mathrm{CDCl}_{3}$ ) 2942 (br, w), 1738 (m), 1158 (m), 1091 (s) $\mathrm{cm}^{-1} ;{ }^{1} \mathrm{H}$ NMR (500 MHz, $\mathrm{CDCl}_{3}$ ) 44.41 (br t, $J=9.0 \mathrm{~Hz}, 2 \mathrm{H}), 4.26(\mathrm{~d}, J=7.5 \mathrm{~Hz}, 2 \mathrm{H}), 3.95$ (dd, $J=5.0,11.0 \mathrm{~Hz}, 2 \mathrm{H}), 3.61(\mathrm{~s}, 6 \mathrm{H}), 3.57$ (s, $6 \mathrm{H}), 3.46(\mathrm{~s}, 6 \mathrm{H}), 3.43(\mathrm{~m}, 4 \mathrm{H}), 3.25(\mathrm{td}, J=5.0,8.0 \mathrm{~Hz}, 2 \mathrm{H}), 3.25(\mathrm{t}, J=11.5 \mathrm{~Hz}, 2 \mathrm{H}), 3.10(\mathrm{t}, J=8.0 \mathrm{~Hz}$, 2H), 3.09 (dd, $J=10.3,11.0 \mathrm{~Hz}, 2 \mathrm{H}), 2.95(\mathrm{dd}, J=7.7,9.1 \mathrm{~Hz}, 2 \mathrm{H}), 2.54(\mathrm{dd}, J=3.5,17.5 \mathrm{~Hz}, 2 \mathrm{H}), 2.41$ $(\mathrm{dd}, J=7.0,17.5 \mathrm{~Hz}, 2 \mathrm{H}), 2.04(\mathrm{dd}, J=5.0,11.5 \mathrm{~Hz}, 2 \mathrm{H}), 1.87(\mathrm{dt}, J=9.0,15.0 \mathrm{~Hz}, 2 \mathrm{H}), 1.66(\mathrm{br} \mathrm{d}, J=$ $15.0 \mathrm{~Hz}, 2 \mathrm{H}), 1.38(\mathrm{~m}, 2 \mathrm{H}), 1.37(\mathrm{q}, J=11.5 \mathrm{~Hz}, 2 \mathrm{H}), 0.96(\mathrm{~d}, J=6.5 \mathrm{~Hz}, 6 \mathrm{H}), 0.96(\mathrm{~d}, J=6.2 \mathrm{~Hz}, 6 \mathrm{H}), 0.82$ $(\mathrm{m}, 2 \mathrm{H}), 0.71(\mathrm{tt}, J=5.0,9.0 \mathrm{~Hz}, 2 \mathrm{H}), 0.33(\mathrm{dt}, J=5.0,8.0 \mathrm{~Hz}, 2 \mathrm{H}), 0.22(\mathrm{dt}, J=5.0,8.0 \mathrm{~Hz}, 2 \mathrm{H}) ;{ }^{13} \mathrm{C} \mathrm{NMR}$ (125 MHz, $\mathrm{CDCl}_{3}$ ) $\square 171.2$ (2C), 105.6 (2C), 85.8 (2C), 84.1 (2C), 83.4 (2C), 79.6 (2C), 77.0-77.2 (4C), 75.1 (2C), 63.4 (2C), 61.0 (2C), 61.0 (2C), 59.0 (2C), 42.7 (2C), 41.5 (2C), 40.9 (2C), 39.4 (2C), 25.0 (2C), 18.7 (2C), $12.8(2 \mathrm{C}), 12.1(2 \mathrm{C}), 11.1(2 \mathrm{C})$; high resolution mass spectrum (ES+) $\mathrm{m} / \mathrm{z} 879.4743\left[(\mathrm{M}+\mathrm{Na})^{+}\right.$; calcd for $\mathrm{C}_{44} \mathrm{H}_{72} \mathrm{O}_{16} \mathrm{Na}^{+}$: 879.4718].

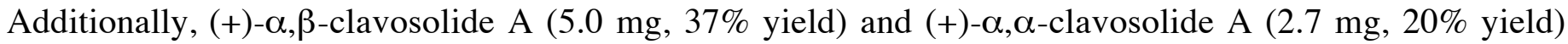
were isolated as byproducts and fully characterized.

${ }^{5}$ Trichloroacetimidate $\mathbf{2 2}$ was prepared using the following sequence:

- $\quad$ permethylation of $\mathrm{D}(+)$-xylose using $\mathrm{Ag}_{2} \mathrm{O}$, MeI; (Walker, H.G.; Gee, M.; McCready, R. M. J. Org. Chem. 1962, 27, 2100.)

- $\quad$ hydrolysis of the methyl acetal using AcOH, TfOH; (Costantino, V.; Barbieri, L. Eur. J. Org. Chem. 2004, 3, 468.)

- $\quad$ trichloroacetimidate formation using $\mathrm{Cl}_{3} \mathrm{CCN}$, DBU; (Kong, F. Carbohydr. Res. 2005, 39.)

The spectroscopic data for all three compounds matched the data reported by Lee and coworkers. (Ref.5 in text) 


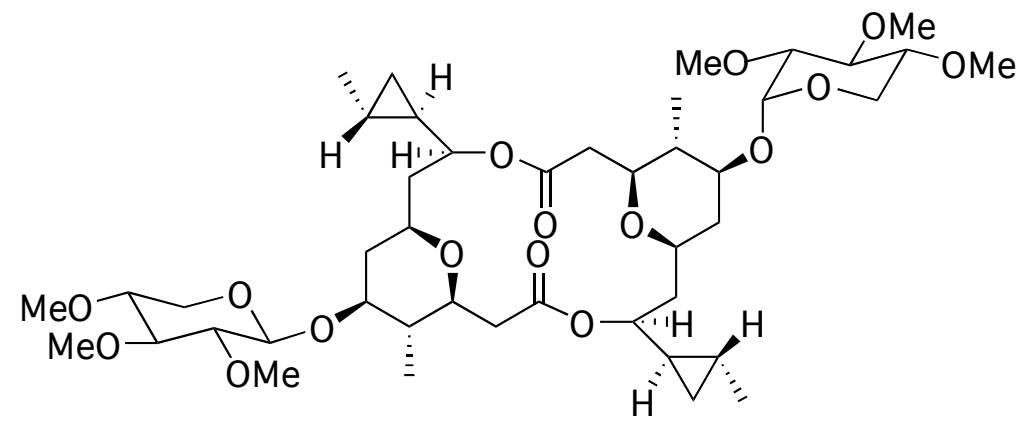

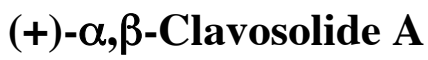

$[\mathrm{D}]_{\mathrm{D}}{ }^{20}+0.3\left(c 0.51, \mathrm{CHCl}_{3}\right)$; IR (thin film, $\left.\mathrm{CDCl}_{3}\right) 2942$ (br, m), $2867(\mathrm{~m}), 1737(\mathrm{~m}), 1158(\mathrm{~m}), 1095(\mathrm{~s})$ $\mathrm{cm}^{-1}$; ${ }^{1} \mathrm{H}$ NMR $\left(500 \mathrm{MHz}, \mathrm{CDCl}_{3}\right) \square 5.05(\mathrm{~d}, J=3.7 \mathrm{~Hz}, 1 \mathrm{H}), 4.43(\mathrm{dt}, J=1.7,8.7 \mathrm{~Hz}, 1 \mathrm{H}), 4.42(\mathrm{dt}, J=1.9$, $8.9 \mathrm{~Hz}, 1 \mathrm{H}), 4.27(\mathrm{~d}, J=7.8 \mathrm{~Hz}, 1 \mathrm{H}), 3.96(\mathrm{dd}, J=4.8,11.5 \mathrm{~Hz}, 1 \mathrm{H}), 3.69(\mathrm{dd}, J=5.6,11.5 \mathrm{~Hz}, 1 \mathrm{H}), 3.63$ $(\mathrm{s}, 3 \mathrm{H}), 3.62(\mathrm{~s}, 3 \mathrm{H}), 3.59(\mathrm{~s}, 3 \mathrm{H}), 3.49(\mathrm{~s}, 6 \mathrm{H}), 3.47(\mathrm{~s}, 3 \mathrm{H}), 3.46-3.44(\mathrm{~m}, 7 \mathrm{H}), 3.25(\mathrm{~m}, 3 \mathrm{H}), 3.17(\mathrm{dd}, J=$ 3.7, $9.7 \mathrm{~Hz}, 1 \mathrm{H}), 3.10(\mathrm{t}, J=8.6 \mathrm{~Hz}, 1 \mathrm{H}), 3.09$ (dd, $J=10.4,11.5 \mathrm{~Hz}, 1 \mathrm{H}), 2.97(\mathrm{dd}, J=7.4,8.9 \mathrm{~Hz}, 1 \mathrm{H})$, $2.58(\mathrm{dt}, J=3.7,17.5 \mathrm{~Hz}, 2 \mathrm{H}), 2.43(\mathrm{dd}, J=6.7,17.5 \mathrm{~Hz}, 2 \mathrm{H}), 2.03(\mathrm{ddd}, J=4.8,4.8,11.9 \mathrm{~Hz}, 2 \mathrm{H}), 1.91(\mathrm{~m}$, 2H), $1.69(\mathrm{ddd}, J=2.6,7.1,15.3 \mathrm{~Hz}, 2 \mathrm{H}), 1.52(\mathrm{~m}, 1 \mathrm{H}), 1.38(\mathrm{q}, J=12.7 \mathrm{~Hz}, 1 \mathrm{H}), 1.37(\mathrm{~m}, 1 \mathrm{H}), 1.22(\mathrm{~m}$, $1 \mathrm{H}), 0.98(\mathrm{~d}, J=5.6 \mathrm{~Hz}, 6 \mathrm{H}), 0.97(\mathrm{~d}, J=6.3 \mathrm{~Hz}, 6 \mathrm{H}), 0.83(\mathrm{~m}, 2 \mathrm{H}), 0.73(\mathrm{tt}, J=5.0,9.0 \mathrm{~Hz}, 2 \mathrm{H}), 0.35(\mathrm{dt}, J$ $=4.8,7.8 \mathrm{~Hz}, 2 \mathrm{H}), 0.23(\mathrm{dt}, J=4.8,8.2 \mathrm{~Hz}, 2 \mathrm{H}) ;{ }^{13} \mathrm{C} \mathrm{NMR}\left(125 \mathrm{MHz}, \mathrm{CDCl}_{3}\right) \square$ 171.2, 171.2, 105.7, 91.9, 85.8, 84.1, 83.4, 82.6, 81.7, 80.1, 79.6, 77.2 (4C), 76.0, 75.1, 74.8, 63.5, 61.2, 61.0 (2C), 60.1, 59.2, 59.0, 58.9, 42.8, 41.7 (2C), 41.4, 40.9, 39.5, 39.4, 36.5, 25.0 (2C), 18.7 (2C), 13.7, 12.8, 12.2, 12.1, 11.1 (2C); high resolution mass spectrum $(\mathrm{ES}+) \mathrm{m} / z, 879.4686$ [(M+Na) ${ }^{+}$; calcd for $\mathrm{C}_{44} \mathrm{H}_{72} \mathrm{O}_{16} \mathrm{Na}^{+}:$879.4718].

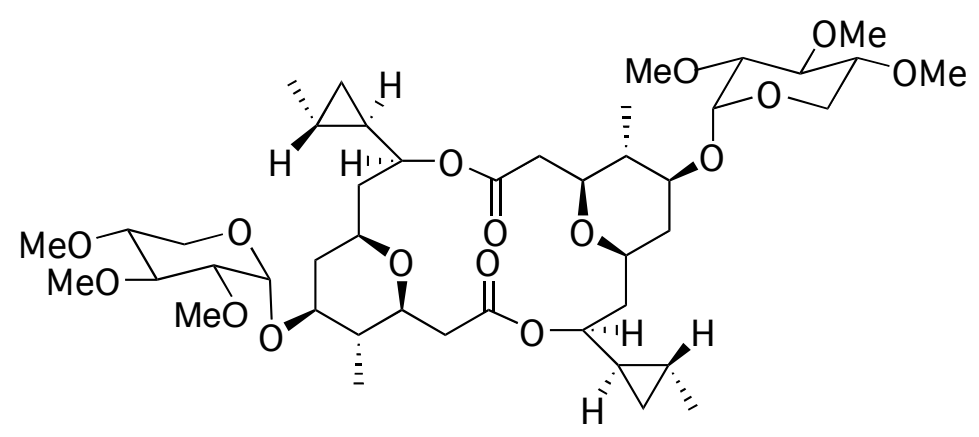

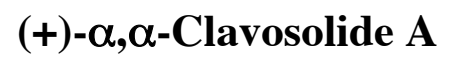

$[\mathrm{C}]_{\mathrm{D}}{ }^{20}+30.0\left(\mathrm{c} \mathrm{0.15}, \mathrm{CHCl}_{3}\right.$ ); IR (thin film, $\left.\mathrm{CDCl}_{3}\right) 2942(\mathrm{br}, \mathrm{m}), 2867(\mathrm{w}), 1737(\mathrm{~m}), 1095(\mathrm{~m}) \mathrm{cm}^{-1} ;{ }^{1} \mathrm{H}$ NMR (500 MHz, $\left.\mathrm{CDCl}_{3}\right) \square 5.05(\mathrm{~d}, J=3.7 \mathrm{~Hz}, 2 \mathrm{H}), 4.43(\mathrm{br} \mathrm{t}, J=9.3 \mathrm{~Hz}, 2 \mathrm{H}), 3.69(\mathrm{dd}, J=5.2,11.2 \mathrm{~Hz}$, 2H), $3.63(\mathrm{~s}, 6 \mathrm{H}), 3.49(\mathrm{~s}, 6 \mathrm{H}), 3.49(\mathrm{~s}, 6 \mathrm{H}), 3.52-3.38(\mathrm{~m}, 10 \mathrm{H}), 3.25(\mathrm{~m}, 2 \mathrm{H}), 3.17(\mathrm{dd}, J=3.7,9.7 \mathrm{~Hz}, 2 \mathrm{H})$, $2.57(\mathrm{dd}, J=3.3,17.5 \mathrm{~Hz}, 2 \mathrm{H}), 2.43(\mathrm{dd}, J=6.3,17.5 \mathrm{~Hz}, 2 \mathrm{H}), 2.02(\mathrm{dd}, J=3.7,12.3 \mathrm{~Hz}, 2 \mathrm{H}), 1.93(\mathrm{dt}, J=$ $8.9,15.3 \mathrm{~Hz}, 2 \mathrm{H}), 1.69$ (d, $J=15.2 \mathrm{~Hz}, 2 \mathrm{H}), 1.53(\mathrm{~m}, 2 \mathrm{H}), 1.22(\mathrm{~m}, 2 \mathrm{H}), 0.98(\mathrm{~d}, J=6.3 \mathrm{~Hz}, 6 \mathrm{H}), 0.97$ (d, $J$ 
$=7.0 \mathrm{~Hz}, 6 \mathrm{H}), 0.84(\mathrm{~m}, 2 \mathrm{H}), 0.73(\mathrm{tt}, J=5.0,9.0 \mathrm{~Hz}, 2 \mathrm{H}), 0.33(\mathrm{dt}, J=4.8,8.9 \mathrm{~Hz}, 2 \mathrm{H}), 0.22(\mathrm{dt}, J=4.8,8.6$ $\mathrm{Hz}, 2 \mathrm{H}) ;{ }^{13} \mathrm{C} \mathrm{NMR}\left(125 \mathrm{MHz}, \mathrm{CDCl}_{3}\right.$ ) $\square 171.2$ (2C), 91.9 (2C), 82.6 (2C), 81.7 (2C), 80.1 (2C), 77.2 (4C), 76.0 (2C), 74.9 (2C), 61.2 (2C), 60.1 (2C), 59.2 (2C), 58.9 (2C), 41.7 (2C), 41.6 (2C), 39.5 (2C), 36.5 (2C), 24.9 (2C), 18.7 (2C), 13.7 (2C), 12.1 (2C), 11.1 (2C); high resolution mass spectrum (ES+) $\mathrm{m} / z 879.4697$ $\left[(\mathrm{M}+\mathrm{Na})^{+}\right.$; calcd for $\left.\mathrm{C}_{44} \mathrm{H}_{72} \mathrm{O}_{16} \mathrm{Na}^{+}: 879.4718\right]$. 